\title{
Dysphonia and its Correlation with Acoustic Voice Parameters
}

\author{
${ }^{1}$ Kavita Sachdeva, ${ }^{2}$ Tanvi Shrivastava
}

\section{ABSTRACT}

Aim: To evaluate the laryngeal causes of dysphonia, correlation of acoustic voice analysis with Indirect laryngoscopic/ endoscopic findings in various voice disorders.

Study design: Hospital based prospective observational study.

Materials and methods: Forty patients attending the ear nose throat (ENT) outpatient department (OPD) at a Tertiary Care Government Hospital in one year, with dysphonia for more than 15 days were selected. History, examination, endoscopy, voice analysis was done. For consensus auditory-perceptual evaluation of voice (CAPE-V), the voice was analyzed under the following parameters: roughness, breathiness, strain, pitch, loudness, overall severity. Scores were given out of 100 . For acoustic analysis, a computer-based software Praat was used based on jitter, shimmer, noise-harmonic ratio and mean pitch.

Result: Benign lesions were most common in adults of age group 21 to 40 years, and malignancy in 41 to 50 years and 61 to 70 years; with male preponderance (4.7:1). Isolated vocal cord palsy $(32.5 \%)$ was the most common lesion presenting with dysphonia, followed by malignancy (25\%). Out of benign lesions, vocal polyp (10\%) and nodule (10\%) were the commonest, with equal incidence. On analyzing the voice, jitter and shimmer were found to be important parameters depicting the perturbation in frequency and amplitude, respectively. These parameters, indirectly, gave an idea about the vibratory motion of the vocal cords. On statistical analysis, jitter and shimmer showed significant direct correlation with the severity of dysphonia (jitter>shimmer). Noise to harmonic ratio (NHR) was raised in a significant number of dysphonic patients, with direct correlation with an increase in jitter. Although a significant relationship between the variation in mean pitch and CAPE $V$ could not be established in this study; instrumental analysis was still useful in documentation and quantification of mean pitch in various dysphonic samples.

Conclusion: Acoustic measurement of voice is a simple yet powerful tool to analyze the patients with dysphonic voice.

Keywords: Acoustic parameters, Consensus auditory-perceptual evaluation of voice (CAPE-V), Dysphonia, Praat Voice analysis.

How to cite this article: Sachdeva K, Shrivastava T. Dysphonia and its Correlation with Acoustic Voice Parameters. Int J Phonosurg Laryngol 2018;8(1):6-12.

${ }^{1}$ Associate Professor, ${ }^{2}$ Senior Resident

1,2Department of Ear, Nose and Throat, Netaji Subhash Chandra Bose Medical College, Jabalpur, Madhya Pradesh, India

Corresponding Author: Tanvi Shrivastava, Senior Resident, Department of Ear, Nose and Throat, Netaji Subhash Chandra Bose Medical College, Jabalpur, Madhya Pradesh, India, e-mail: tanvishrivastava07@gmail.com
Source of support: Nil

Conflict of interest: None

AIM

To evaluate the laryngeal causes of dysphonia and find a correlation of acoustic voice analysis with indirect laryngoscopic/endoscopic findings in various voice disorders.

\section{INTRODUCTION}

A voice disorder occurs when voice quality, pitch, and loudness differ or are inappropriate for an individual's age, gender, cultural background, or geographic location (Aronson and Bless; Boone, McFarlane, Von Berg, and Zraik; Lee, Stemple, Glaze, and Kelchner).

- Organic: Voice disorders that are physiological in nature and result from alterations in respiratory, laryngeal, or vocal tract mechanisms

- Structural: Organic voice disorders (OVD) that result from physical changes in the voice mechanism (e.g., alterations in vocal fold tissues such as edema or vocal nodules; structural changes in the larynx due to aging)

- Neurogenic: Organic voice disorders (OVD) that result from problems with the central or peripheral nervous system innervation to the larynx that affect the functioning of the vocal mechanism (e.g., vocal tremor, spasmodic dysphonia, or paralysis of vocal folds)

- Functional: Voice disorders that result from the improper or inefficient use of the vocal mechanism when the physical structure is normal (e.g., vocal fatigue; muscle tension dysphonia or aphonia; diplophonia; ventricular phonation)

\section{VOICE ANALYSIS}

\section{Subjective}

The CAPE-V uses continuous visual analog scales for judgments of six parameters of voice: overall severity, roughness, breathiness, strain, pitch, and loudness. The perceptual evaluation of voice is considered to be an essential aspect of the conventional voice diagnostics that is of primary relevance to most voice-disordered patients and provides a global measure of vocal performance 
readily available to all clinicians (Orlikoff, Dejonckere, Dembowski et al., 1999).

\section{Instrumental/Objective}

Using the computer-based software Praat, which is free and easy, it supports the clinician to rely on objective scientific data. It measures four basic parameters:

\section{Jitter, Shimmer, Harmonic to Noise Ratio, Pitch}

Jitter refers to cycle-to-cycle, short-term perturbation in the fundamental frequency of the voice. It is the relative evaluation of the very short-term variability of the pitch within the analyzed voice sample. Jitter is one of the main measures for micro-instability in vocal fold vibrations. Even tiny growths on vocal folds, such as polyps, may influence frequency perturbation. According to the available literature, jitter increases as control over laryngeal muscle tone becomes coarser or as the number of active motor units decreases. ${ }^{1}$

The shimmer is a cycle-to-cycle, short-term perturbation in the amplitude of voice. It increases with poor and inconsistent contact between the vocal fold edges.

Incomplete closure of the glottis caused by polyp leads to an air leakage, which is acoustically characterized as noise. The NHR is the average ratio of the inharmonic energy to the harmonic spectral energy. It includes contributions from both perturbations of amplitude and frequency. $^{2}$

Epidemiologic studies of the prevalence and risk factors of voice disorders in the general population are relatively rare. Moreover, the small numbers of studies that exist are characterized by substantial variability in reported prevalence estimates, ranging from $0.65 \%$ to $29.9 \%$ in the general population. ${ }^{3,4}$ In spite of high prevalence, in most government-run hospitals in India, just an auditory perception of voice [CAPE-V or grade, roughness, breathiness, asthenia, strain (GRBAS)] is done without any quantification of the auditory data. This lack of standard voice measure compromises the evaluation of different treatments and their outcomes and becomes particularly important in this day of medical liability. To effectively evaluate their outcome a quantitative measure of voice function providing relevant information is needed. So an attempt has been made to study and assess the quantitative parameters of voice using the software Praat, concerning various disorders and to highlight its implication in the present scenario.

\section{STUDY DESIGN}

Hospital based prospective observational study.

\section{MATERIALS AND METHODS}

Forty patients attending the ear, nose and throat (ENT) outpatient department (OPD), Netaji Subhash Chandra Bose Medical College, Jabalpur, in 1 year, with dysphonia for more than 15 days were selected. Patients who could produce prolonged phonation for at least 3 seconds, were selected (as required for voice analysis). Detailed history, general and ENT examination was done.

Indirect/direct laryngoscopy was done. Site, size, the extent of the lesion (if present), laterality, the mobility of vocal cords were noted. A biopsy was taken, when required.

Subjective and objective voice analysis was done using CAPE-V and Praat respectively.

Voice was analyzed under the following parameters:

- Roughness

- Breathiness

- Strain

- Pitch

- Loudness

- Overall severity Scores were given out of 100

Stimuli given were:

- Sustained vowels for 3 to 5 seconds each

- Reading

- Spontaneous speech

\section{Additional Features}

Tremor, pitch instability, diplophonia, falsetto, wet/ gurgly or other relevant terms.

\section{Objective Analysis}

A computer-based software Praat was used. The voice samples were analyzed quantitatively based on:

- Jitter

- Shimmer

- Noise-harmonic ratio

- Mean pitch

The normative values vary according to the software. Normative for Praat are as follows:
Jitter: $\quad \leq 1.040 \%$
Shimmer: $\quad \geq 3.810 \%$
NHR: $\quad<1$

$\begin{array}{lll} & \text { Adult males } & \text { Adult females } \\ \text { Mean pitch: } & 128 \mathrm{~Hz} & 225 \mathrm{~Hz} \\ \text { Minimum pitch: } & 85 \mathrm{~Hz} & 155 \mathrm{~Hz} \\ \text { Maximum pitch: } & 196 \mathrm{~Hz} & 334 \mathrm{~Hz}\end{array}$

Voice therapy was given to these patients and was kept on follow-up.

Lesions which did not respond to voice therapy were subjected to MLS. 
RESULT

\section{Age Group}

- Voice disorders were found in a wide age group of 15 to 80 years. Benign lesions were most common in adults of age group 21 to 40 years. Malignant lesions were more commonly seen in the age group of 41 to 50 years and 61 to 70 years.

\section{Sex}

- Males were more commonly affected than females in benign as well as malignant lesions with a ratio of 4.7:1. Functional dysphonia with hoarseness was more commonly seen in females (Graph 1).

- All the patients belonged to a low socio-economic status with an equal preponderance of rural and urban background.

\section{Occupation}

- Total $30 \%$ of patients in the study were farmers. Benign lesions were more commonly seen in occupations which required excessive voice usage such as singers and housewives with young children at home (Table 1).

- The predisposing factors were tobacco smoking and chewing, alcohol and voice abuse. Vocal abuse was an important predisposing factor in benign lesions like vocal polyp and nodule. Smoking and alcohol had a strong correlation with the development of malignancy (Table 2).

\section{Symptomatology}

Dysphonia is any impairment of the voice or difficulty in speaking. All 40 patients presented with impairment of voice. Details of the impairment in voice were noted. A maximum number of patients presented with the change

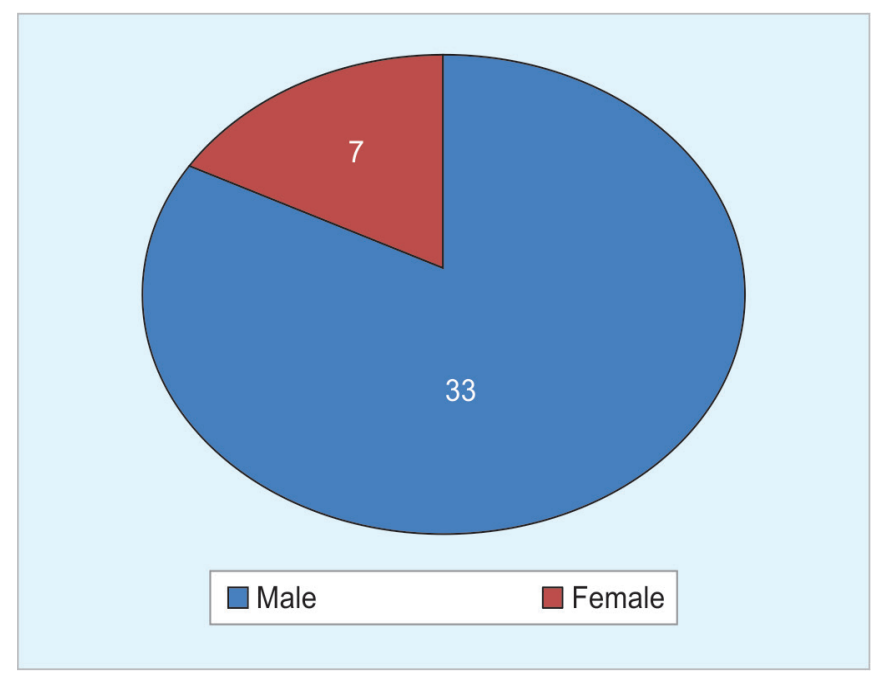

Graph 1: Sex distribution
Table 1: Occupation wise distribution

\begin{tabular}{lll}
\hline Occupation & Frequency & Percent (\%) \\
\hline Army & 1 & 2.5 \\
Asha worker & 1 & 2.5 \\
Bidi maker & 1 & 2.5 \\
Farmer & 12 & 30 \\
House wife & 3 & 7.5 \\
Labour & 9 & 22.5 \\
Mechanic & 1 & 2.5 \\
Rickshaw driver & 1 & 2.5 \\
Student & 5 & 12.5 \\
Security guard & 1 & 2.5 \\
Singer & 1 & 2.5 \\
Supervisor & 1 & 2.5 \\
Truck driver & 2 & 5 \\
Vendor & 1 & 2.5 \\
Total & 40 & 100 \\
\hline
\end{tabular}

Table 2: Predisposing factors for development of dysphonia

\begin{tabular}{lll}
\hline Predisposing factors & Frequency & Percent (\%) \\
\hline Smoking & 23 & 57.5 \\
Tobacco chewing & 11 & 27.5 \\
Alcohol & 9 & 22.5 \\
Voice abuse & 16 & 40 \\
History of trauma & 4 & 10 \\
\hline
\end{tabular}

in the quality of voice which was described as rough/ hoarse/strangulated/breathy/strained by the patients, $5 \%$ of complaint. Another $5 \%$ of patients complained of frequent voice breaks while phonation. Painful phonation was seen in $7.5 \%$ of patients, whereas vocal fatigue was seen in all the patients. Weak voice or inability to increase loudness was seen in $7.5 \%$ of patients (Tables 3 and 4).

Throat pain was seen in $70 \%$ of patients. Pain or irritation in throat was felt either continuous or only while speaking. It was also associated with vocal fatigue.It was the predominant symptom in laryngeal tuberculosis (TB).

Cough was present in $47.5 \%$ of patients. In most of the cases, there was a history of bouts of cough followed by fluid intake. This could be attributed to aspiration due to improper coordination during phases of swallowing. One hundred percent of cases of TB laryngitis complained of cough, which was expectorant, and occasionally blood tinged. A cough was also seen in patients with gastroesophageal reflux disease (GERD).

Dysphagia or difficulty in swallowing was seen in $32.5 \%$ cases, most of them had malignant lesions involving the laryngopharynx. Dyspnoea was seen in $25 \%$ of cases, most of them being cases of malignancy. Chest pain was present in $7.5 \%$ of cases. Earache was seen in $5 \%$ of cases. 
Dysphonia and its Correlation with Acoustic Voice Parameters

\begin{tabular}{lll}
\hline \multicolumn{3}{c}{ Table 3: Frequency of different symptoms } \\
\hline Symptoms & Frequency & Percent (\%) \\
\hline Dysphonia & 40 & 100 \\
Throat pain & 28 & 70 \\
Cough & 19 & 47.5 \\
Difficulty in swallowing & 13 & 32.5 \\
Dyspnoea & 10 & 25 \\
Chest pain & 3 & 7.5 \\
Earache & 2 & 5 \\
Neck swelling & 0 & 0 \\
\hline
\end{tabular}

Table 5: Types of lesion in IDL and DL

\begin{tabular}{lll}
\hline & Frequency & $\begin{array}{l}\text { Percent } \\
(\%)\end{array}$ \\
\hline Malignancy & 10 & 25 \\
Isolated vocal cord palsy & 13 & 32.5 \\
Nodule & 4 & 10 \\
Polyp & 4 & 10 \\
Reinke's edema & 3 & 7.5 \\
Laryngeal TB & 1 & 2.5 \\
No lesion/functional dysphonia & 5 & 12.5 \\
\hline
\end{tabular}

\section{Examination}

- On indirect and direct laryngoscopy, $32.5 \%$ of patients presenting with dysphonia had isolated unilateral vocal cord palsy. This was followed by malignancy, which was seen in $25 \%$ of patients. Benign lesions were seen in $20 \%$ of patients. Functional dysphonia was seen in $12.5 \%$ of patients which also included two patients with puberphonia. Laryngeal tuberculosis was a rare disorder (Table 5).

- Benign lesions were most commonly seen in anterior 1/3rd of vocal cords (Table 6).

- Majority of cases of malignancy presented with stage III. All of the cases were squamous cell carcinoma, mostly moderately differentiated (Table 7).

- Voice analysis was done for each patient, using CAPE-V and Praat, for subjective and acoustic assessment of voice, respectively.

\begin{tabular}{lll}
\multicolumn{3}{c}{ Table 4: Detailed symptomatology of dysphonia } \\
\hline & Frequency & Percent (\%) \\
\hline Voice quality & 38 & 95 \\
Painful phonation & 3 & 7.5 \\
Weak voice & 3 & 7.5 \\
Change in pitch & 2 & 5 \\
Voice breaks & 2 & 5 \\
Vocal fatigue & 40 & 100 \\
\hline
\end{tabular}

Table 6: Sites of benign lesions

\begin{tabular}{llllll}
\hline \multirow{5}{*}{$\begin{array}{l}\text { Type of } \\
\text { lesion }\end{array}$} & Site of lesion & & & \\
\cline { 2 - 6 } & commisure & Ant & Middle & Post & Post \\
\hline Polyp & 2 & 2 & - & - & - \\
Nodule & - & 3 & 1 & - & - \\
Laryngeal TB & - & - & - & - & 1 \\
\hline
\end{tabular}

Table 7: Stages in malignant lesions

\begin{tabular}{lll}
\hline Stage & No. of cases & Percent (\%) \\
\hline I & 1 & 10 \\
II & 2 & 20 \\
III & 7 & 70 \\
IV & 0 & 0 \\
Total & 10 & 100 \\
\hline
\end{tabular}

- Severity was marked on a scale of 100 and was categorized as mild, moderate and severe. Quality was noted as roughness, breathiness, strained. Abnormal loudness and pitch were also recorded (Tables 8 and 9).

\section{VOICE ANALYSIS IN VARIOUS DISORDERS}

\section{Jitter and Shimmer}

\section{Vocal Nodule and Polyp}

According to CAPE-V, all the patients had a 'rough' voice, with mild to moderate dysphonia in most cases. There was one patient who had 'severe' dysphonia; which could be attributed to the large size of the polyp.

Table 8: Subjective-audio perceptual analysis (CAPE-V)

\begin{tabular}{|c|c|c|c|c|c|c|c|c|}
\hline \multirow[b]{2}{*}{ Diagnosis } & \multirow[b]{2}{*}{ Total } & \multicolumn{3}{|c|}{ Severity of dysphonia } & \multicolumn{4}{|c|}{ Quality of voice } \\
\hline & & Mild & Moderate & Severe & Normal & Roughness & Breathy & Strained \\
\hline Isolated vocal cord palsy & 13 & 5 & 7 & 1 & 0 & 11 & 2 & 0 \\
\hline Malignancy & 10 & 4 & 3 & 3 & 0 & 9 & 1 & 0 \\
\hline Vocal polyp & 4 & 1 & 2 & 1 & 0 & 2 & 2 & 0 \\
\hline Vocal nodule & 4 & 1 & 3 & 0 & 0 & 3 & 0 & 1 \\
\hline Reinke's edema & 3 & 2 & 1 & 0 & 0 & 3 & 0 & 0 \\
\hline Idiopathic & 3 & 0 & 3 & 0 & 0 & 2 & 0 & 1 \\
\hline Puberphonia & 2 & 2 & 0 & 0 & 1 & 0 & 0 & 1 \\
\hline TB larynx & 1 & 0 & 1 & 0 & 0 & 1 & 0 & 0 \\
\hline
\end{tabular}




\begin{tabular}{|c|c|c|c|c|c|c|c|c|c|c|}
\hline & \multirow{2}{*}{$\begin{array}{l}\text { Total no of } \\
\text { cases }\end{array}$} & \multicolumn{2}{|c|}{ Jitter } & \multicolumn{2}{|c|}{ Shimmer } & \multicolumn{2}{|l|}{$H N R$} & \multicolumn{3}{|c|}{ Mean pitch } \\
\hline & & I & $N$ & $I$ & $N$ & $A b n$ & $N$ & $N$ & $I$ & $D$ \\
\hline Isolated VC palsy & 13 & 8 & 5 & 2 & 11 & 8 & 5 & 1 & 9 & 3 \\
\hline Malignancy & 10 & 6 & 4 & 3 & 7 & 6 & 4 & 1 & 8 & 1 \\
\hline Vocal nodule & 4 & 2 & 2 & 1 & 3 & 3 & 1 & 0 & 2 & 2 \\
\hline Vocal polyp & 4 & 2 & 2 & 1 & 3 & 3 & 1 & 0 & 2 & 2 \\
\hline Reinke's edema & 3 & 3 & 0 & 2 & 1 & 3 & 0 & 0 & 2 & 1 \\
\hline Idiopathic & 3 & 3 & 0 & 2 & 1 & 3 & 0 & 0 & 0 & 3 \\
\hline Puberphonia & 2 & 0 & 2 & 0 & 2 & 2 & 0 & 0 & 2 & 0 \\
\hline TB larynx & 1 & 1 & 0 & 0 & 1 & 1 & 0 & 0 & 0 & 1 \\
\hline
\end{tabular}

Perturbation in frequency or jitter was increased in $50 \%$ of patients.

Pertubation in amplitude or shimmer was increased only in $25 \%$ of patients, and $75 \%$ of patients were within the normal range of shimmer.

Patients with jitter and shimmer within the normal range were good candidates for voice therapy. The lesion either subsided or reduced in size significantly on giving voice therapy which significantly affected the further management outcome in such patients. It should be noted that this categorization of patients could be achieved only after doing the acoustic analysis, and not when the samples were assessed solely based on CAPE-V.

\section{Isolated Vocal Cord Palsy}

Jitter-62.5\% patients showed significantly raised jitter, whereas shimmer was increased in $18.8 \%$ patients. Most of the patients had 'mild to 'moderate' 'rough' voice. Two patients who had incomplete compensation by the contralateral vocal cord had a significant phonatory gap, which added the 'breathy' component to the quality of voice. Considering the patient compliance, there was a satisfactory response to voice therapy in patients who followed the regular regime of the therapy. Cases which did not show sufficient response were later planned for thyroplasty.

\section{Malignancy}

Consensus auditory-perceptual evaluation of voice (CAPE-V)-'roughness' was the predominant quality of voice as perceived according to CAPE- $\mathrm{V}$. The severity ranged from mild to severe; more advanced and extensive lesions exhibiting 'severe' dysphonic voice. Patients with phonatory gap showed a 'breathy' component along with roughness in the voice samples.

Jitter was increased in $60 \%$ of patients with malignant lesions. Increase in severity (CAPE V) was directly proportional to the increase in jitter.

Shimmer was increased in $30 \%$ of patients with malig- nant lesions. $70 \%$ of patients did not show any significant increase in shimmer.

\section{Reinke's Edema}

These patients presented with 'mild' to 'moderate' dysphonia. Jitter was increased in all the patients with shimmer being affected in $66.6 \%$ of patients. These patients did not respond well to voice therapy alone. Cessation of smoking was helpful in a few cases.

\section{Functional Dysphonia}

Patients without any organic lesion were categorized as functional dysphonia.

Idiopathic: On perceptual analysis, their voice samples were categorized as 'moderate' 'breathy/strained.'Increase in jitter $(100 \%)$ and shimmer(66.6\%) was seen.

Puberphonia: It was seen in adolescent males, with an increase in pitch with typical values for jitter as well as shimmer.

These patients of functional dysphonia showed the best response to voice therapy and laryngeal massage. Additionally, a psychiatric evaluation was done in patients with puberphonia.

\section{SUMMARY}

Jitter and shimmer are important parameters depicting the perturbation in frequency and amplitude, respectively. These parameters, indirectly, give an idea about the vibratory motion of the vocal cords. On statistical analysis, jitter and shimmer showed significant direct corelation with the severity of dysphonia (Jitter>> shimmer).

Hoarseness due to benign lesions of vocal cords, with near to normal jitter and shimmer, was seen to respond well to voice therapy alone; as compared to the patients with increased jitter and shimmer which needed microlaryngoscopic surgery at a later stage along with voice therapy. 


\section{Noise-harmonic Ratio}

It includes contributions from both perturbations of amplitude and frequency. Thus, it provides a compilation of jitter and shimmer. The ratio is $<1$ in a normal voice.

\section{Vocal Nodule/Polyp}

A total of $75 \%$ of patients with benign vocal lesions, had raised NHR.

\section{Malignancy}

A total of $60 \%$ of patients had NHR $>1$. It was associated with an increase in jitter as well. Patients with normal NHR had jitter too within the normal range.

\section{Isolated Vocal Cord Palsy}

A total of $61.5 \%$ patients had increased NHR. These patients had increased jitter too.

\section{Reinke's Edema and Functional Dysphonia}

The NHR was $>1$ in all the patients with functional dysphonia and Reinke's edema.

\section{Summary}

The NHR was raised in a significant number of dysphonic patients, with direct correlation with an increase in jitter.

\section{Mean Pitch}

Mean pitch was found to be increased in $62.5 \%$ of the dysphonic voices. A total of $25 \%$ cases had a decreased mean pitch. Although a significant relationship between the variation in mean pitch and CAPE-V could not be established in this study; instrumental analysis was still useful in documentation and quantification of mean pitch in various dysphonic samples.

\section{STATISTICAL ANALYSIS}

The statistical analysis showed the correlation between the severity of dysphonia (CAPE-V) and Praat, among various voice disorders. Pearson correlation was applied, and value of severity was compared with jitter, shimmer, HNR mean pitch in different voice disorder. Voice disorders have a strong positive correlation with values of severity and jitter $(r=0.733)$, shimmer $(r=0.743)$, NHR $(\mathrm{r}=0.829)$ respectively. It means as the values in severity index of CAPE-V increase, the value among jitter, shimmer, NHR positively increase.

There are a statistically significant correlations between severity and jitter, shimmer, NHR values. This means the increase in the severity of dysphonia significantly relate to the increase in Jitter, Shimmer, and NHR. A direct correlation between the severity of dysphonia and mean pitch $(r=0.440)$ could not be established in this study.

\section{To Summarise}

On statistical analysis, voice analysis using Praat proved to be an essential tool for quantitative assessment of dysphonia, which further helped in deciding the management as well as assessing the effectiveness of the ongoing treatment.

\section{DISCUSSION}

Hoarseness is the most common presenting complaint, in benign as well as malignant lesions involving the vocal cords. ${ }^{5}$ It is postulated that repeated trauma due to vocal abuse and other mechanical factors causes denudation of the mucosa over vocal cords, resulting in granulation tissue. Hoarseness occurs due to incomplete closure of glottis by this mass of granulation tissue. In the study by Hegde et al., hoarseness was present in $100 \%$ patients with benign lesions of the larynx, followed by a cough and throat pain. Frequent voice breaks were seen in patients with functional dysphonia. Vocal fatigue was seen in all the patients and was more severe in patients with more extensive lesions and more phonatory gap. Dysphonia was continuous and persistent in most of the cases except patients with puberphonia, who complained of an intermittent change in voice. Throat pain and cough could be attributed to aspiration in cases of impaired vocal cord mobility. Malignant lesions involving the laryngopharynx complained of dysphagia. Vocal Polyp: In the present study, four cases of vocal polyps $(10 \%)$ were seen. Vocal polyps, as well as vocal nodules, were the most common benign lesions in this study, which correlates with the study by Hegde et al., where he studied 42 patients with benign lesions, with vocal polyps being the commonest (40\%).

Vocal Nodule: In the present series, there were four cases $(10 \%)$ of vocal nodules. The size ranged from 1-2 mm. Marc Bouchayer et al. in their series showed an incidence of $28.76 \%$.

Reinke's Edema: Reinke's space is the subepithelial matrix of the vocal fold mucosa composed of elastin, collagen, and other extracellular proteins; it is also known as the superficial lamina propria. Reinke's edema is the accumulation of fluid in this space. The condition is fairly common and constitutes about $10 \%$ of benign laryngeal lesions as per previous studies. In the present study, it was present in $7.5 \%$ of patients. 
Tuberculous Laryngitis: Ormerod stated that TB laryngitis is prevalent in male aged between 20 to 30 years. TB laryngitis is a rare entity now since the introduction of effective anti tubercular treatment (ATT). There was a single case of TB laryngitis and was in accordance to the finding stated by Ormerod above. Biopsy was the diagnostic procedure of choice which showed typical features of tuberculosis on histopathological examination.

Malignancy of larynx: Out of 40 cases, 25\% of patients had laryngeal cancer. Out of these, $70 \%$ of cases were glotto-supraglottic with extension to pre/para epiglottic space. $10 \%$ of cases were restricted to glottic region. Cases with extensive lesion who were not able to undergo voice analysis ( which requires prolonged phonation for 3 seconds) were not taken in the study.

In a study by Mirjana et al., the results showed that jitter $(\%)$, shimmer (\%), fundamental frequency variation (vF0), voice turbulence index (VTI), pitch perturbation quotient (PPQ), amplitude perturbation quotient (APQ), and NHR values significantly differentiate the patients with vocal fold polyps from a control group without any pathological changes in the larynx.

The present study shows significant variation in jitter, shimmer, and HNR with increasing severity of symptoms in various voice disorders. ${ }^{6}$ Correlation between mean pitch and severity was not significant. Thus, both perceptual and acoustic parameters are essential to asses the voice across multiple voice disorders.

\section{CONCLUSION}

In the developing nations of the world, the need felt today is not just quality healthcare, but quality healthcare at an affordable cost. Although the perceptual evaluation of the patients' voice remains one of the most critical evaluation tools, it is not devoid of drawbacks. Lack of trained listeners, subjective bias and inter-listener variations in the assessment are some of the most commonly faced difficulties. ${ }^{7}$ Therefore, the need was felt to devise a system that would be free of the above fallacies.
A standard measure of voice is necessary for the current healthcare environment. Since voice is a multidimensional function, perceptual and objective measures are necessary to "grade" voice. ${ }^{8}$ Acoustic measurement of voice is a simple yet powerful tool to analyze the patients with dysphonic voice.

Analysis of voice, using CAPE-V and Praat, is a promising tool-

- For quantitative assessment of dysphonia to analyze the pre and post-treatment values.

- To asses the efficacy of the ongoing treatment to decide the further management.

\section{REFERENCES}

1. Von Berg, California State University, Chico. Richard I. Zraick, University of Arkansas for Medical Sciences; Voice and Voice Therapy, 9th Edition.

2. Martin D, Fitch J, Wolfe V. Pathological voice type and the acoustic prediction, of severity./Speech Hear Res. 1995;38:765771.

3. Roy N, Merrill RM, Gray SD, Smith EM. Voice disorders in the general population: prevalence, risk factors, and functional impact. Laryngoscope. 2005 Nov;115(11):1988-1995.

4. Roy N, Merrill RM, Thibeault S, Parsa RA, Gray SD, Smith EM. Prevalence of voice disorders in teachers and the general population. Journal of Speech, Language, and Hearing Research. 2004 Apr 1;47(2):281-293.

5. Hegde MC, Kamath MP, Bhojwani K, Peter R, Babu PR. Benign lesions of larynx-A clinical study. Indian Journal of Otolaryngology and Head \& Neck Surgery. 2005;57(1):35-38.

6. Wolfe V, Fitch J, Martin D. Acoustic measures of dysphonic severity across and within voice types. Folia Phoniatrica et Logopaedica. 1997;49:292-299.

7. Bhuta T, Patrick L, Garnett JD. Perceptual evaluation of voice quality and its correlation with acoustic measurements. Journal of voice. 2004 Sep 1;18(3):299-304.

8. Agarwal PK, Biswas KD, Dutta M, Ghatak S, Sen I. Quantitative Assessment of voice by Acoustic Analysis in patients undergoing Microlaryngeal Surgery for vocal cord nodules. Bengal Journal of Otolaryngology and Head Neck Surgery. 2013 Jun 30;21(1):15-16. 